Original Research

\title{
Association between symptomatic intracranial atherosclerotic disease and the integrity of the circle of Willis
}

\author{
Yilan $\mathrm{Xu}^{1}$, Dongye $\mathrm{Li}^{2}$, Wei Dai ${ }^{3,4}$, Zhuozhao Zheng $^{1, *, \dagger}$, Xihai Zhao ${ }^{1,5, *, \dagger}$ \\ ${ }^{1}$ Department of Radiology, Beijing Tsinghua Changgung Hospital, School of Clinical Medicine, Tsinghua University, 102218 Beijing, China \\ ${ }^{2}$ Department of Radiology, Sun Yat-sen Memorial hospital, Sun Yat-sen Universitye, 510123 Cuangzhou, Cuangdong, China \\ ${ }^{3}$ Department of Neurology, Fourth Medical Center of Chinese PLA General Hospital, 100048 Beijing, China \\ ${ }^{4}$ Department of Neurology, Chinese PLA Ceneral Hospital \& Medical School of Chinese PLA, 100039 Beijing, China \\ ${ }^{5}$ Department of Biomedical Engineering, Center for Biomedical Imaging Research, Tsinghua University School of Medicine, 100084 Beijing, China \\ *Correspondence: zzza00509@btch.edu.cn (Zhuozhao Zheng); xihaizhao@tsinghua.edu.cn (Xihai Zhao) \\ ${ }^{\dagger}$ These authors contributed equally.
}

DOI:10.31083/j.jin2101002

This is an open access article under the CC BY 4.0 license (https://creativecommons.org/licenses/by/4.0/).

Submitted: 26 August 2021 Revised: 26 October 2021 Accepted: 19 November 2021 Published: 20 January 2022

The relationship between the severity of intracranial atherosclerotic disease and the circle of Willis integrity is unclear. In this brief report, we investigate the associations between symptomatic intracranial atherosclerotic disease and the integrity of the circle of Willis. Patients with symptomatic intracranial atherosclerosis were enrolled and underwent intracranial artery magnetic resonance vessel wall imaging and time-of-flight angiography. The presence or absence of an intracranial atherosclerotic plaque and its maximum wall thickness and stenosis were evaluated. The presence or absence of the A1 segment of the bilateral anterior cerebral arteries (from the internal carotid artery to the anterior communicating artery segment is called anterior cerebral artery A1 segment), and anterior communicating artery, the $\mathrm{P} 1$ segment of the bilateral posterior cerebral arteries (The P1 segment of the posterior cerebral artery is a horizontally outward segment), and bilateral posterior communicating arteries were determined. The associations of the intracranial plaque features with the integrity of the circle of Willis were analyzed. Of the 110 recruited subjects ( $57.2 \pm 11.1$ years; $65 \%$ males), 51 had intracranial plaques, and 44 had stenosis. In patients with bilateral $A 1$ and $P 1$ segments (n $=85$ ), intracranial stenosis was more severe in patients with an anterior communicating artery than those without an anterior communicating artery $(19.7 \% \pm 21.7 \%$ vs. $1.4 \% \pm 3.3 \%, p=0.046)$. In patients with bilateral $\mathrm{A}_{1}$ and $\mathrm{P}_{1}$ segments and an anterior communicating artery $(n=79)$, intracranial stenosis was more severe in patients with posterior communicating arteries than those without posterior communicating arteries $(27.9 \% \pm 23.7 \%$ vs. $13.5 \% \pm 17.9 \%, p=0.007)$. The odds ratio of intracranial stenosis was 1.214 (95\% confidence interval $(\mathrm{Cl}), 1.054--1.398 ; p=0.007)$ in discriminating for the presence of posterior communicating arteries in patients with bilateral $A 1$ and P1 segments and an anterior communicating artery after adjusting for confounding factors. The severity of intracranial atherosclerosis was independently associated with the presence of posterior communicating arteries in patients with a complete anterior part of the circle of Willis.

\section{Keywords}

Intracranial artery; Atherosclerosis; Circle of Willis; Magnetic resonance imaging; Vessel wall; Neurovascular

\section{Introduction}

Intracranial atherosclerosis is the main cause of ischemic stroke in an Asian population, divided into symptomatic and asymptomatic types. Symptomatic intracranial atherosclerosis refers to ischemic stroke or transient ischemic attack occurring in the stenosis area of the supplying artery [1]. It is well established that the progression of the intracranial atherosclerotic disease will decrease blood perfusion and increase the risk of cerebral ischemic events [2-6]. During the progression of atherosclerotic cerebrovascular disease, the compensatory system can be initiated through the collateral circulation to preserve perfusion and stabilize cerebral blood flow [7-11]. As the primary collateral pathway, the circle of Willis (COW) connects the bilateral anterior circulations via the anterior communicating artery $(\mathrm{ACoA})$ or the anterior and posterior circulations via the posterior communicating arteries (PCoAs). The integrity of the COW usually represents the capability of compensation when there is a decline in cerebral blood flow [12,13]. Since atherosclerosis is a systemic disease that frequently involves multiple vascular beds (including the contralateral carotid artery and basilar artery), knowledge of the dominant communicating arteries is important for developing treatment strategies in patients diagnosed with atherosclerotic diseases multiple vascular beds.

Limited studies have demonstrated that the severity of atherosclerotic carotid disease is associated with the presence of collateral flow in the communicating arteries. Hartkamp et al. [14] demonstrated that patients with internal carotid artery (ICA) obstruction were more likely to have complete anterior parts of the COW, such as the presence of bilateral 
A1 segments and an ACoA, or an entirely complete COW. Researchers also found that the stenosis of an ICA obstruction was correlated with cross-flow in the ACoA $[15,16]$. However, the association between the severity of intracranial artery atherosclerosis and the integrity of the COW (particularly the status of the PCoAs) remains unclear.

It is hypothesized that intracranial atherosclerotic disease may activate the communicating arteries in certain orders that integrate bilateral circulations and/or anterior and posterior circulations as the disease severity increases. This study investigates the associations between the severity of intracranial atherosclerotic disease and the integrity of communicating arteries in the COW using three-dimensional (3D) magnetic resonance (MR) vessel wall imaging.

\section{Methods}

\subsection{Study sample}

In this study, patients with recent (within 2 weeks) ischemic stroke or transient ischemic attack (TIA) in anterior circulation determined by either imaging findings or clinical diagnosis were continuously recruited. All patients underwent MR imaging. The exclusion criteria were as follows: (1) cardiogenic stroke, hemorrhagic stroke; (2) patients who failed to complete MR examination due to contraindication, including pacemaker, artificial valve, nerve stimulator, eyeball foreign body and convulsion with a high fever. Clinical information (age, sex, body mass index, cholesterol level, and blood pressure) was recorded. Each patient collected history of hypertension, diabetes mellitus, smoking, hyperlipidemia, stroke, transient ischemic attack, and coronary heart disease.

\subsection{MR imaging}

The MR imaging was conducted on a 3.0T MR scanner (Achieva TX, Philips Healthcare; Best, the Netherlands) with a custom-designed 36-channel neurovascular coil. Intracranial arteries were imaged by acquiring images of the vessel wall and angiography with three-dimensional motionsensitizing driven equilibrium rapid gradient-echo sequence (3D MERGE), 3D T1-volume isotropic turbo spine-echo acquisition (VISTA), and 3D time-of-flight (TOF). The extracranial carotid arteries were imaged by TOF MR angiography. The parameters for 3D MERGE were as follows: fast field echo; repeat time/echo time, 9.2/4.3 ms; flip angle, $6^{\circ}$; field of view, $4.0 \times 16 \times 25 \mathrm{~cm}^{3}$; spatial resolution, $0.8 \times$ $0.8 \times 0.8 \mathrm{~mm}^{3}$. The parameters for 3D T1-VISTA were as follows: turbo spin echo; repeat time/echo time, 700/21 ms; field of view, $4.5 \times 20 \times 20 \mathrm{~cm}^{3}$; spatial resolution, $0.6 \times$ $0.6 \times 0.6 \mathrm{~mm}^{3}$. The parameters for $3 \mathrm{D}$ TOF were as follows: fast field echo; repeat time/echo time, $25 / 3.5 \mathrm{~ms}$; field of view, $4.5 \times 20 \times 20 \mathrm{~cm}^{3}$; spatial resolution, $0.7 \times 0.7 \times$ $1.4 \mathrm{~mm}^{3}$.

\subsection{Image review}

Two experienced radiologists interpreted the MR images for consensus using an MR workstation (Extended MR WorkSpace 2.6.3.4; Best, the Netherlands). Intracranial atherosclerosis was defined as eccentric wall thickening occurring in the following arterial segments of anterior circulation: internal carotid artery $(\mathrm{C} 3-\mathrm{C} 7), \mathrm{M} 1 \mathrm{segment}$ of the middle cerebral artery (MCA) and A1 segment of the anterior cerebral artery (ACA). The vessel wall images determined the presence or absence of intracranial artery plaques. The maximum wall thickness (Max WT) and stenosis of the corresponding intracranial atherosclerotic plaque were measured when a plaque was present. Luminal stenosis was measured on the maximum intensity projection images of TOF MR angiography (MRA) utilizing criteria for warfarinaspirin symptomatic intracranial disease (WASID) [17] and North American Symptomatic Carotid Endarterectomy Trial NASCET [18] for intracranial arteries and carotid arteries, respectively. The presence or absence of the A1 segment of the bilateral anterior cerebral arteries, an ACoA, the P1 segment of the bilateral posterior cerebral arteries, and bilateral PCoAs was evaluated on the TOF MRA images.

\subsection{Statistical analysis}

The Shapiro-Wilk normality test tests the normality of the data distribution. Continuous variables were reported as the mean and standard deviation, and discrete variables were described as percentages. The clinical characteristics were compared between patients with and without an ACoA or PCoAs using the Mann-Whitney or chi-square test (where appropriate). When there were multiple plaques in the intracranial arteries of one patient, the most severe plaque burden (including Max WT and stenosis) among all plaques was selected for statistical analysis. It is well evidenced that the severity of plaque burden was associated with plaque vulnerability at the patient level. Thus, an atherosclerotic plaque with the most severe burden may represent the most vulnerable plaque among multiple lesions. For patients with bilateral A1 and P1 segments, the Max WT and stenosis of the intracranial plaques were compared between subjects with and without an ACoA or PCoAs using the non-parametric Mann-Whitney U test. Univariate and multivariate logistic regressions were performed to determine the odds ratios and corresponding 95\% confidence intervals (CIs) of the intracranial artery plaque, Max WT, and stenosis in discriminating the presence of an ACoA or PCoAs. The probability of intracranial artery stenosis in predicting the presence of an ACoA or PCoAs before and after adjusting for confounding factors was calculated using C-statistical analysis. A pvalue $<0.05$ was considered statistically significant. All statistical analyses were performed using SPSS 16.0 (SSPS Inc.; Chicago, IL, USA).

\section{Results \\ 3.1 Patient characteristics}

One hundred ten patients (mean age, $57.2 \pm 11.1$ years) were recruited from January 2013 to March 2014. Of these patients, 72 (65.5\%) were male, 74 (67.3\%) had hypertension, 77 (70\%) had hyperlipidemia, 58 (52.7\%) had a history of smoking, and 35 (31.8\%) had a history of diabetes. Ta- 
Table 1. Clinical characteristics of the study population.

\begin{tabular}{|c|c|c|c|c|c|c|c|}
\hline & \multicolumn{7}{|c|}{ Mean $\pm \mathrm{SD}$, or $\mathrm{n}(\%)$} \\
\hline & All patients & *ACoA (+) & *ACoA (-) & \multirow{2}{*}{$p$} & $\# \mathrm{PCoA}(+)$ & \#PCoA (-) & \multirow{2}{*}{$p$} \\
\hline & $(\mathrm{n}=110)$ & $(\mathrm{n}=79)$ & $(\mathrm{n}=6)$ & & $(\mathrm{n}=34)$ & $(\mathrm{n}=45)$ & \\
\hline Age, years & $57.2 \pm 11.1$ & $56.7 \pm 12.2$ & $56.8 \pm 6.5$ & 0.976 & $56.5 \pm 11.3$ & $56.8 \pm 13.0$ & 0.938 \\
\hline Male sex & $72(65.5)$ & $52(65.8)$ & $2(33.3)$ & 0.185 & $24(70.6)$ & $28(62.2)$ & 0.438 \\
\hline BMI, $\mathrm{kg} / \mathrm{m}^{2}$ & $25.3 \pm 3.1$ & $25.3 \pm 3.2$ & $25.4 \pm 2.3$ & 0.951 & $25.4 \pm 3.0$ & $25.3 \pm 3.3$ & 0.864 \\
\hline Smoking & $58(52.7)$ & $42(53.2)$ & $2(33.3)$ & 0.423 & $18(52.9)$ & $24(53.3)$ & 0.972 \\
\hline Hypertension & $74(67.3)$ & $48(60.8)$ & $5(83.3)$ & 0.402 & $21(61.8)$ & $27(60)$ & 0.874 \\
\hline Hyperlipidemia & $77(70)$ & $55(69.6)$ & $5(83.3)$ & 0.756 & $23(67.6)$ & $32(71.1)$ & 0.939 \\
\hline $\mathrm{LDL}, \mathrm{mmol} / \mathrm{L}$ & $2.86 \pm 1.13$ & $2.94 \pm 1.22$ & $2.16 \pm 0.93$ & 0.074 & $3.20 \pm 1.59$ & $2.75 \pm 0.80$ & 0.323 \\
\hline $\mathrm{HDL}, \mathrm{mmol} / \mathrm{L}$ & $1.12 \pm 0.36$ & $1.10 \pm 0.35$ & $0.99 \pm 0.10$ & 0.635 & $1.19 \pm 0.44$ & $1.03 \pm 0.25$ & 0.152 \\
\hline $\mathrm{TC}, \mathrm{mmol} / \mathrm{L}$ & $4.53 \pm 1.15$ & $4.51 \pm 1.16$ & $4.00 \pm 1.28$ & 0.313 & $4.63 \pm 1.40$ & $4.42 \pm 0.96$ & 0.444 \\
\hline $\mathrm{TG}, \mathrm{mmol} / \mathrm{L}$ & $1.60 \pm 0.89$ & $1.64 \pm 0.95$ & $1.62 \pm 0.85$ & 0.951 & $1.46 \pm 0.70$ & $1.77 \pm 1.09$ & 0.373 \\
\hline DM & $35(31.8)$ & $22(28.2)$ & $4(66.7)$ & 0.071 & $11(32.4)$ & $11(25.0)$ & 0.474 \\
\hline Statin use & $82(74.5)$ & $56(70.9)$ & $6(100)$ & 0.184 & $23(67.6)$ & $33(73.3)$ & 0.582 \\
\hline History of stroke & $62(56.4)$ & $38(48.1)$ & $6(100)$ & 0.026 & $16(47.1)$ & $22(48.9)$ & 0.872 \\
\hline History of CHD & $16(14.5)$ & $12(15.2)$ & $0(0)$ & 0.588 & $5(14.7)$ & $7(15.6)$ & 0.917 \\
\hline
\end{tabular}

BMI, body mass index; LDL, low-density lipoprotein; HDL, high-density lipoprotein; TC, total cholesterol; TG, total glyceride; DM, Diabetes mellitus; CHD, coronary heart disease. ${ }^{*}$ For patients with the presence of bilateral A1 and P1 segments. \#For patients with bilateral A1 and P1 segments and ACoA.

ble 1 shows the clinical characteristics of the study population among the different COW integrity groups. For patients with bilateral A1 and P1 segments, no significant differences were found in all clinical characteristics except for a history of stroke ( $48.1 \%$ vs. $100 \%, p=0.026)$ between patients with and without an ACoA. Similarly, for patients with bilateral A1 and P1 segments and an ACoA, no significant differences were found in all clinical characteristics between patients with and without PCoAs (all $p>0.05$ ).

\subsection{Association between integrity of the circle of Willis and intracranial atherosclerosis}

Of the 110 patients, 51 (46.4\%) and 44 (40\%) were found to have atherosclerotic plaques and stenosis in the intracranial arteries, respectively. Of all 51 patients with intracranial plaques, 75 plaques were detected, and 45 plaques were located in C3-C7 segments of intracranial internal carotid arteries, 23 plaques were located in the M1 segment of MCA and 7 plaques located in the A1 segment of ACA.

The presence of bilateral A1 segments, bilateral P1 segments, and both bilateral A1 and P1 segments were observed in 100 (90.9\%), 92 (83.6\%), and 85 (77.3\%) patients, respectively. In addition, 91 (82.7\%) patients had an ACoA, and 58 (52.7\%) patients had PCoAs. The whole anterior part of the COW (presence of bilateral A1 segments and an ACoA) and the whole posterior part of the COW (presence of bilateral P1 segments and PCoAs) were found in 91 (82.7\%) and 15 (13.6\%) patients, respectively. An entirely complete COW was found in $13(11.8 \%)$ patients.

For patients with both bilateral A1 and P1 segments ( $\mathrm{n}=$ 85), the intracranial stenosis in patients with an ACoA was significantly more severe than in patients without an ACoA $(19.7 \% \pm 21.7 \%$ vs. $1.4 \% \pm 3.3 \%, p=0.046)$ (Table 2$)$. For patients with both bilateral A1 and P1 segments and an ACoA $(\mathrm{n}=79)$, the intracranial stenosis $(27.9 \% \pm 23.7 \%$ vs. $13.5 \%$ $\pm 17.9 \%, p=0.007)$ and the presence of plaques (76.5\% vs. 48.9\%, $p=0.013$ ) in patients with PCoAs were significantly more severe than in those patients without PCoAs (Table 2). For patients who had intracranial plaques, the Max WT of the intracranial artery was similar to patients with and without an ACoA $(p=0.268)$ or PCoAs $(p=0.748)$ (Table 2$)$. An example of a patient with severe intracranial artery stenosis and the presence of bilateral A1 and P1 segments, an ACoA, and PCoAs is shown in Fig. 1.

For patients with bilateral A1 and P1 segments, the presence of an ACoA was not significantly associated with the presence of plaques, Max WT, and stenosis before and after adjusting for the clinical confounding factors of age, sex, body mass index, hypertension, smoking, diabetes mellitus, hyperlipidemia, history of stroke and the stenosis degree of extracranial carotid atherosclerosis (all $p>0.05$, Table 3 ). The logistic regression analysis results for patients with bilateral A1, P1, and an ACoA are shown in Table 4. The odds ratio of intracranial stenosis with increments of $5 \%$ was 1.174 (95\% CI, 1.051-1.313; $p=0.005$ ) and 1.214 (95\% CI, 1.054$1.398 ; p=0.007$ ) in discriminating for the presence of PCoAs before and after adjusting for the above confounding factors. The odds ratio of the presence of plaques was 3.398 (95\% CI, 1.269-9.095; $p=0.015$ ) and 4.374 (95\% CI, 1.145-16.701; $p$ $=0.031)$ in discriminating for the presence of PCoAs before and after adjusting for the above confounding factors.

\section{Discussion}

The results demonstrated a significant difference in stenosis of the intracranial arteries between patients with and 
Table 2. Intracranial plaque features in patients with different integrity of COW.

\begin{tabular}{|c|c|c|c|c|c|c|}
\hline & \multicolumn{6}{|c|}{ Mean $\pm \mathrm{SD}$, or $\mathrm{n}(\%)$} \\
\hline & ${ }^{*} \mathrm{ACoA}(+)$ & ${ }^{*} \mathrm{ACoA}(-)$ & \multirow{2}{*}{$p$} & \#PCoA (+) & \#PCoA (-) & \multirow{2}{*}{$p$} \\
\hline & $(\mathrm{n}=79)$ & $(\mathrm{n}=6)$ & & $(\mathrm{n}=34)$ & $(\mathrm{n}=45)$ & \\
\hline Presence of plaque & $48(60.8)$ & $3(50)$ & 0.679 & $26(76.5)$ & $22(48.9)$ & 0.013 \\
\hline Max WT, mm & $2.2 \pm 0.8$ & $1.7 \pm 0.8$ & 0.268 & $2.2 \pm 0.8$ & $2.2 \pm 0.7$ & 0.748 \\
\hline Stenosis, \% & $19.7 \pm 21.7$ & $1.4 \pm 3.3$ & 0.046 & $27.9 \pm 23.7$ & $13.5 \pm 17.9$ & 0.007 \\
\hline
\end{tabular}

*For patients with presence of bilateral A1 and P1 segments. \#For patients with presence of bilateral

A1 and P1 segments and ACoA.
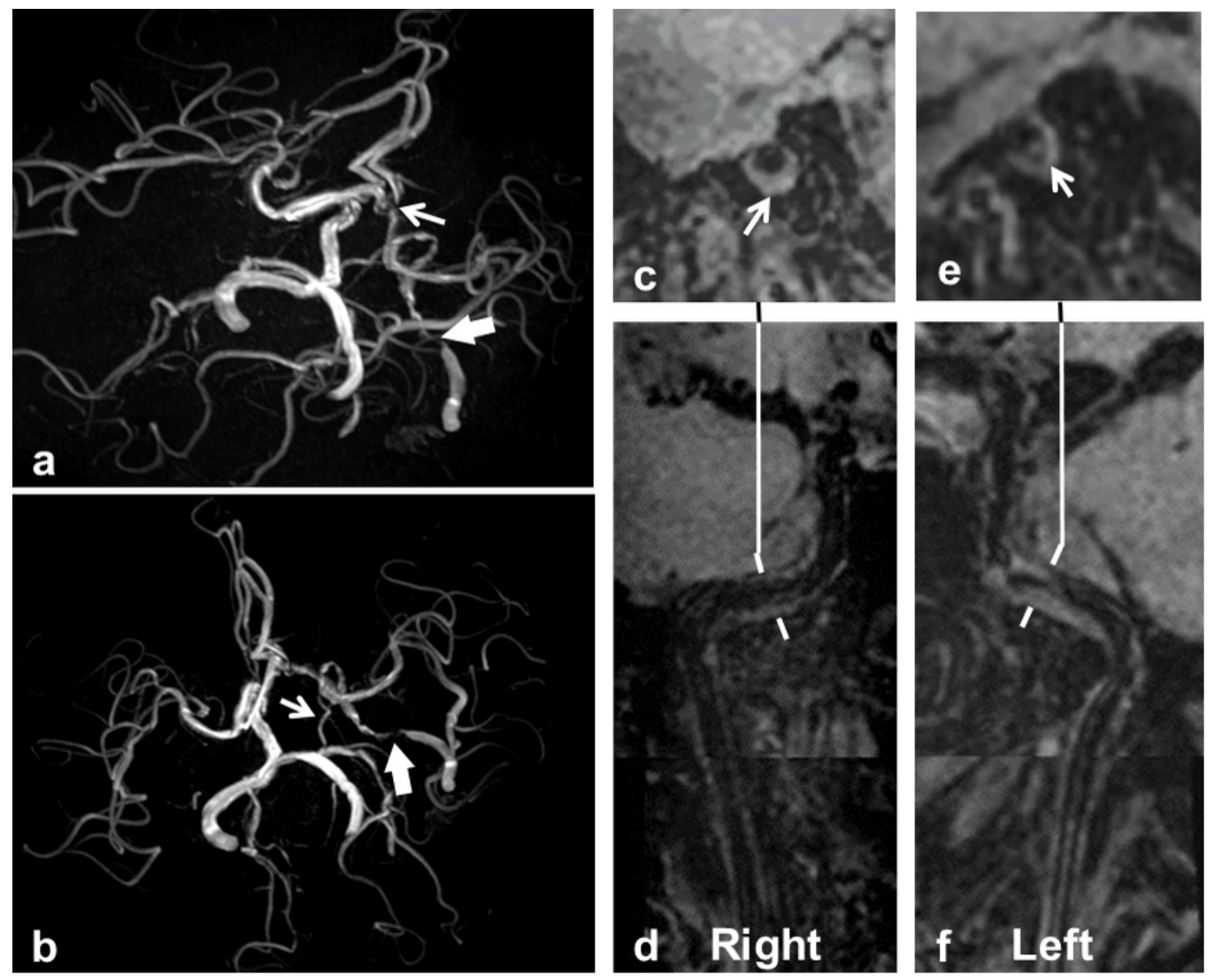

Fig. 1. MR images of a 60-year-old man with severe intracranial artery stenosis who also had an anterior and posterior communicating artery (ACoA and PCoA, respectively). (a) and (b) represent the MIP images of TOF MRA. ACoA and left PCoA are present (thin white arrows in a and b), and severe stenosis in the C2 segment of the left internal carotid artery was observed (thick arrows in a and b). (d) and (f) represent the curved reconstructed images of MERGE. Two plaques in the C2 segments of the bilateral internal carotid arteries (arrows in c and e) are shown.

without an ACoA and PCoAs. It was also determined that characteristics of intracranial artery atherosclerosis, including the presence of plaques, and stenosis, were independently associated with PCoAs in patients with bilateral A1 and P1 segments and an ACoA. The findings indicate that the severity of intracranial artery atherosclerosis may be an independent indicator of the integrity of the COW.

A complete COW was only found in $11.8 \%$ of the patients. The data showed that $82.7 \%$ and $13.6 \%$ of the patients had complete structures of the anterior and posterior parts of the COW, respectively. Two previous studies reported similar results regarding the prevalence of a complete anterior part of the COW (78\%-80.95\%) [19,20]. In the present study, the prevalence of whole complete and complete posterior structures of the COW was slightly lower than that reported in the two former studies; one study reported a prevalence of $20.9 \%$ and $16.6 \%$, respectively, whereas the other reported a prevalence of $21.0 \%$ and $33.0 \%$, respectively. These differences may be due to this study's smaller sample size. In addition, the findings are consistent with a previous study for the presence of bilateral A1 (this study's data vs. previous data: 90.9\% vs. $95.0 \%$ ) and bilateral P1 (this study's data vs. previous data: $83.6 \%$ vs. $86.7 \%$ ) segments.

In patients diagnosed with bilateral A1 and P1 segments, intracranial stenosis was more severe in patients with the presence of an ACoA than those without, and a marginal as- 
Table 3. Association between atherosclerosis and presence of ACoA.

\begin{tabular}{lcccccc}
\hline & \multicolumn{5}{c}{ Presence of ACoA* } \\
\cline { 2 - 7 } & \multicolumn{3}{c}{ Univariate regression } & \multicolumn{3}{c}{ Multivariate regression\# } \\
\cline { 2 - 7 } & OR & $95 \%$ CI & $p$ & OR & $95 \%$ CI & $p$ \\
\hline Presence of plaque & 1.548 & $0.294-8.166$ & 0.606 & 0.455 & $0.045-4.618$ & 0.505 \\
Max WT, mm & 2.588 & $0.406-16.494$ & 0.314 & 1.823 & $0.132-25.274$ & 0.654 \\
Stenosis $\dagger, \%$ & 1.692 & $0.792-3.617$ & 0.175 & 2.087 & $0.768-5.675$ & 0.149 \\
\hline
\end{tabular}

*For patients with the presence of bilateral A1 and P1 segments. \#Adjustment for age, gender, BMI, hypertension, smoking, diabetes mellitus, hyperlipidemia, history of stroke and stenosis of the extracranial carotid artery. $+W i$ ith increment of $5 \%$.

Table 4. Association between atherosclerosis and presence of PCoA.

\begin{tabular}{lcccccc}
\hline & \multicolumn{5}{c}{ Presence of PCoA* } \\
\cline { 2 - 7 } & \multicolumn{3}{c}{ Univariate regression } & \multicolumn{3}{c}{ Multivariate regression\# } \\
\cline { 2 - 7 } & OR & $95 \%$ CI & $p$ & OR & $95 \%$ CI & $p$ \\
\hline Presence of plaque & 3.398 & $1.269-9.095$ & 0.015 & 4.374 & $1.145-16.701$ & 0.031 \\
Max WT, mm & 0.883 & $0.421-1.851$ & 0.742 & 1.108 & $0.433-2.832$ & 0.831 \\
Stenosis $\dagger$ \% & 1.174 & $1.051-1.313$ & 0.005 & 1.214 & $1.054-1.398$ & 0.007 \\
\hline *For patients with the presence of bilateral A1, P1 segments and ACOA. \#Adjustment for age, \\
gender, BMI, hypertension, smoking, diabetes mellitus, hyperlipidemia, history of stroke and \\
stenosis of the extracranial carotid artery. $\dagger$ With increment of 5\%.
\end{tabular}

sociation was found between the stenosis and the presence of an ACoA. The results are consistent with previous studies [14, 21]. Hartkamp et al. [14] demonstrated that patients with an ICA obstruction had a significantly higher percentage of entirely complete COW configurations (55\% vs. $36 \%, p=$ 0.02 ) and complete anterior configurations ( $88 \%$ vs. $68 \%, p=$ 0.002 ) when compared with control subjects. Furthermore, patients with an ICA occlusion were found to have a higher prevalence of collateral flow through the anterior COW.

Kablak-Ziembicka et al. [21] reported that the ACoA was instrumental in maintaining collaterals within the COW. Similar results were found in patients with carotid occlusion [22]. Zhu et al. [23] demonstrated that the flow of ACoA is the most sensitive index to the morphology change of ipsilateral ICA in-vitro study. These results revealed that communicating arteries (as collateral pathways of cerebral blood flow) might play a key role in the compensational capability of cerebral arteries, and their presence may be associated with the severity of stenosis.

In addition to the ACoA, collateral circulation through the PCoAs was reported in previous studies [14, 21, 24, 25]. However, the ACoA was more likely to be present than PCoAs when unilateral ICA stenosis occurred [14, 21, 25]. This study's data demonstrated that for patients with bilateral A1 and P1 segments and an ACoA, intracranial stenosis was more severe in those with PCoAs. Hartkamp et al. [14] also found that patients with an ICA obstruction had a significantly higher percentage of complete posterior COW configurations than the control group. As such, it is suggested that the COW tends to be complete, and collateral circulation seems to integrate from the anterior to posterior communicating arteries with an increase in the severity of intracranial stenosis. This hypothesis can be evidenced in part by previous in vitro or model studies [23, 26, 27]. For example, investigators reported the reduction of ipsilateral blood flow from the ICA, which is most sensitively detected by the flow of ACoA. The collateral function of the PCoAs will not be activated until severe stenosis in the ICA occurs.

This study had several limitations. First, this was a crosssectional study lacking the dynamic changes of the COW with the progression of cerebral vascular stenosis. Second, the integrity of the COW was analyzed with TOF MRA that is insensitive to blood flows with slow velocities; therefore, the absence of communicating arteries may have been overestimated. Contrast CTA or MRA are superior to TOF MRA for evaluating the morphology of cerebral vasculatures because of a contrast agent. TOF MRA measures luminal stenosis, and the criteria used to define luminal stenosis are originally digital subtraction angiography (DSA). Third, the logistic regression analysis could not represent the general population since the conditions of Circle of Willis are complicated. Utilizing one regression model could not stratify different conditions of Circle of Willis. Fourth, although the degree of extracranial carotid atherosclerosis slightly affected the association between intracranial plaques and COW morphology, the carotid stenosis in this study population was dominantly mild to moderate, suggesting further investigation by including individuals with a broader range of luminal stenosis in future studies for minimizing patient selection bias. In addition, only the most severe plaque burden was assessed in patients with multiple intracranial plaques, ignoring differences in atherosclerotic burden among patients. 


\section{Conclusions}

In conclusion, the severity of intracranial artery atherosclerosis is independently associated with the presence of PCoAs for patients with a complete anterior part of the COW. Future prospective studies are warranted to determine the causal relationship between the increase of severity of intracranial artery atherosclerosis disease and the time course of integration of collateral circulation.

\section{Author contributions}

YLX searched the literatures, analyzed the data and drafted the manuscripts. DYL and WD collected and analyzed the data. ZZZ and XHZ provide supervision, analyzed the data and edited the manuscript.

\section{Ethics approval and consent to participate}

The local ethics committee approved the study, and these patients obtained informed consent. The Ethics Committee of our hospital reviewed and approved this study (No. 20110017), and all involved patients provided written consent forms.

\section{Acknowledgment}

We thank Dongxiang Xu from University of Washington in USA for the management of MR imaging data.

\section{Funding}

This study was supported by grants of Beijing $\mathrm{Mu}$ nicipal Administration of Hospitals' Youth Program (QML20180902), National Natural Science Foundation of China (81801694, 81771825), Beijing Municipal Science and Technology Commission (D171100003017003), and Ministry of Science and Technology of China (2017YFC1307904).

\section{Conflict of interest}

The authors declare no conflict of interest.

\section{References}

[1] Ma YH, Leng XY, Dong Y, Xu W, Cao X, Ji X, et al. Risk factors for intracranial atherosclerosis: a systematic review and metaanalysis. Atherosclerosis. 2019; 281: 71-77.

[2] Pu Y, Lan L, Leng X, Wong LK, Liu L. Intracranial atherosclerosis: from anatomy to pathophysiology. International Journal of Stroke. 2017; 12: 236-245.

[3] Qureshi AI, Caplan LR. Intracranial atherosclerosis. The Lancet. 2014; 383: 984-998.

[4] Kim BS, Chung PW, Park KY, Won HH, Bang OY, Chung CS, et al. Burden of Intracranial Atherosclerosis is Associated with LongTerm Vascular Outcome in Patients with Ischemic Stroke. Stroke. 2017; 48: 2819-2826.

[5] Zhang X, Zhou Y, Zhang S, Ding W, Lou M. Cerebral blood flow evaluation of intensive rosuvastatin therapy in stroke/transient ischemic attack patients with intracranial arterial atherosclerotic stenosis study: Rationale and design. Brain and Behavior. 2017; 7: e00689.

[6] Hoshino T, Sissani L, Labreuche J, Ducrocq G, Lavallée PC, Meseguer E, et al. Prevalence of Systemic Atherosclerosis Burdens and Overlapping Stroke Etiologies and their Associations with Long-term Vascular Prognosis in Stroke with Intracranial Atherosclerotic Disease. JAMA Neurology. 2018; 75: 203-211.

[7] Kluytmans M, van der Grond J, van Everdingen KJ, Klijn CJ, Kappelle LJ, Viergever MA. Cerebral hemodynamics in relation to patterns of collateral flow. Stroke. 1999; 30: 1432-1439.

[8] Shuaib A, Butcher K, Mohammad AA, Saqqur M, Liebeskind DS Collateral blood vessels in acute ischaemic stroke: a potential therapeutic target. The Lancet Neurology. 2011; 10: 909-921.

[9] Liebeskind DS, Cotsonis GA, Saver JL, Lynn MJ, Cloft HJ, Chimowitz MI, et al. Collateral circulation in symptomatic intracranial atherosclerosis. Journal of Cerebral Blood Flow and Metabolism. 2011; 31: 1293-1301.

[10] Liebeskind DS. Understanding blood flow: the other side of an acute arterial occlusion. International Journal of Stroke. 2007; 2: 118-120.

[11] Liebeskind DS, Cotsonis GA, Saver JL, Lynn MJ, Turan TN, Cloft $\mathrm{HJ}$, et al. Collaterals dramatically alter stroke risk in intracranial atherosclerosis. Annals of Neurology. 2011; 69: 963-974.

[12] Kennedy McConnell F, Payne S. The Dual Role of Cerebral Autoregulation and Collateral Flow in the Circle of Willis after Major Vessel Occlusion. IEEE Transactions on Bio-Medical Engineering. 2017; 64: 1793-1802.

[13] Hoksbergen AW, Legemate DA, Ubbink DT, de Vos HJ, Jacobs NJ. Influence of the collateral function of the circle of Willis on hemispherical perfusion during carotid occlusion as assessed by transcranial colour-coded duplex ultrasonography. European Journal of Vascular and Endovascular Surgery. 1999; 17: 486-492.

[14] Hartkamp MJ, van Der Grond J, van Everdingen KJ, Hillen B, Mali WP. Circle of Willis collateral flow investigated by magnetic resonance angiography. Stroke. 1999; 30: 2671-2678.

[15] Bisschops RHC, Klijn CJM, Kappelle LJ, van Huffelen AC, van der Grond J. Collateral flow and ischemic brain lesions in patients with unilateral carotid artery occlusion. Neurology. 2003; 60: 14351441.

[16] Hendrikse J, Hartkamp MJ, Hillen B, Mali WP, van der Grond J. Collateral ability of the circle of Willis in patients with unilateral internal carotid artery occlusion: border zone infarcts and clinical symptoms. Stroke. 2001; 32: 2768-2773.

[17] Samuels OB, Joseph GJ, Lynn MJ, Smith HA, Chimowitz MI. A standardized method for measuring intracranial arterial stenosis. American Journal of Neuroradiology. 2000; 21: 643-646.

[18] Eliasziw M, Smith RF, Singh N, Holdsworth DW, Fox AJ, Barnett HJ. Further comments on the measurement of carotid stenosis from angiograms. North American Symptomatic Carotid Endarterectomy Trial (NASCET) Group. Stroke. 1994; 25: 24452449.

[19] Jalali Kondori B, Azemati F, Dadseresht S. Magnetic resonance angiographic study of anatomic variations of the circle of Willis in a population in Tehran. Archives of Iranian Medicine. 2017; 20: 235-239.

[20] Naveen SR, Bhat V, Karthik GA. Magnetic resonance angiographic evaluation of circle of Willis: a morphologic study in a tertiary hospital set up. Annals of Indian Academy of Neurology. 2015; 18: 391-397.

[21] Kabłak-Ziembicka A, Przewłocki T, Pieniazek P, Musiałek P, Motyl R, Rudko R, et al. Evaluation of cerebral circulation in patients with significant carotid artery stenosis. Kardiologia Polska. 2005; 63: 381-390.

[22] Miralles M, Dolz JL, Cotillas J, Aldoma J, Santiso MA, Giménez A, et al. The role of the circle of Willis in carotid occlusion: assessment with phase contrast MR angiography and transcranial duplex. European Journal of Vascular and Endovascular Surgery. 1995; 10: 424-430.

[23] Zhu G, Yuan Q, Yang J, Yeo J. Experimental study of hemodynamics in the Circle of Willis. Biomedical Engineering Online. 2015; 14: S1-S10.

[24] Guo ZN, Sun X, Liu J, Sun H, Zhao Y, Ma H, et al. The Impact of Variational Primary Collaterals on Cerebral Autoregulation. Frontiers in Physiology. 2018; 9: 759. 
[25] Hoksbergen AW, Fülesdi B, Legemate DA, Csiba L. Collateral configuration of the circle of Willis: transcranial color-coded duplex ultrasonography and comparison with postmortem anatomy. Stroke. 2000; 31: 1346-1351.

[26] Zhu G, Yuan Q, Yang J, Yeo JH. The role of the circle of Willis in internal carotid artery stenosis and anatomical variations: a computational study based on a patient-specific three-dimensional model. BioMedical Engineering Online. 2015; 14: 107.

[27] Alastruey J, Parker KH, Peiró J, Byrd SM, Sherwin SJ. Modeling the circle of Willis to assess the effects of anatomical variations and occlusions on cerebral flows. Journal of Biomechanics. 2007; 40: 1794-1805. 